\title{
A 19 year old with heavy vaginal bleeding; unlikely diagnosis of uterine carcinsarcoma
}

\section{Andrew L. Atkinson*, Paulina Osial, Michael Manuel}

Department of Obstetrics and Gynecology, Jersey Shore University Medical Center, Neptune New Jersey, USA

Received: 3 April 2013

Accepted: 27 April 2013

\section{*Correspondence:}

Dr. Andrew L. Atkinson,

E-mail: A.AtkinsonMD@gmail.com

(C) 2013 Atkinson AL et al. This is an open-access article distributed under the terms of the Creative Commons Attribution License, which permits unrestricted use, distribution, and reproduction in any medium, provided the original work is properly cited.

\begin{abstract}
Uterine carcinosarcoma is a rare malignancy that has a high recurrence rate after treatment with very poor long term prognosis. Women are diagnosed usually over the age of 50, with most cases occurring between the sixth and seventh decade of life. We present a rare case of uterine carcinosarcoma diagnosed a nineteen year old after having a dilation and curettage for heavy vaginal bleeding. The patient went on to have a total abdominal hysterectomy with transposition of the ovaries as well as adjuvant treatment with chemotherapy.
\end{abstract}

Keywords: Uterine Carcinosarcoma, Mixed Mullerian Tumor, Teenager, Ovarian Transposition

\section{INTRODUCTION}

Uterine carcinosarcoma was first described in 1852 , wherein it was recognized as a mixed mesodermal tumor that was initially called "enchondroma". ${ }^{1}$ Today it is believed that carcinosarcomas have a monoclonal origin from a common multidirectional progenitor stem cell. ${ }^{1}$ Carcinosarcomas though rare, representing less than $5 \%$ of all uterine tumors, account for $16.4 \%$ of all deaths caused by uterine malignancy. ${ }^{2}$ Over 10 percent of patients with carcinosarcoma will present with metastatic disease, and 60 percent will have extrauterine disease. ${ }^{3}$ Women are diagnosed usually over the age of 50 , with most cases occurring between the sixth and seventh decade, with a median age of 62 years. ${ }^{4,5}$ Risk factors for the development of carcinosarcoma are similar to those of endometrial carcinoma, including nulliparity, advanced age, obesity, exposure to exogenous estrogens, and long-term use of tamoxifen. ${ }^{6,7}$ Women with uterine carcinosarcoma may present with a classical triad of pain, vaginal bleeding, and a rapidly enlarging uterus. Of these, vaginal bleeding is the most common presenting sign., ${ }^{8,9}$ The current recommended treatment is definitive surgical staging with total abdominal hysterectomy (TAH), bilateral salpingooophorectomy (BSO), pelvic lymphadenectomy, and para-aortic lymph-node sampling with peritoneal washings followed by chemotherapy. ${ }^{10}$ Recurrences in uterine carcinosarcomas occur in over half $(53 \%)$ of patients after primary surgical and adjuvant therapy. ${ }^{11}$ Even in early-stage disease, rates of recurrence are reported between $47 \%-64 \%$ and up to $80 \%$ of these will be associated with distant metastases. ${ }^{12,13}$ Specific factors that increase the risk of recurrence include patient age, adnexal spread, metastases to the lymph nodes, tumour size, lymphatic-vascular space involvement, histologic grade, cell type, peritoneal cytologic findings, and the depth of invasion of the primary tumor. ${ }^{10}$ Over the past forty years despite evolving and advancing therapeutic regimes, prognosis remains poor, with no significant improvement in survival or recurrence rates. ${ }^{12}$

\section{CASE REPORT}

A 19 year old nulligravid Caucasian female with a body mass index of 31 , presents to the emergency room of a community medical center complaining of heavy vaginal 
bleeding for the past 4 months. She reports that she had normal monthly menses since menarche at age 13 , but became amenorrheic from the age of 16 on. Four months prior to presentation, she started experiencing daily spotting that progressed to heavy vaginal bleeding with passage of large clots. The patient had no medical conditions and only underwent a laparoscopic cholecystectomy at age 15 . She had no significant family history and denied cigarette, alcohol or illicit drug use. The patient in the emergency department was found to be pale, orthostatic and had a baseline heart rate of 128 . On pelvic exam, 30cc's of clotted blood was found in the vaginal vault, but no active bleeding from the cervical os was noted. A complete blood count was done and it was found that her hemoglobin was 6.1 , and hematocrit 24.6. She subsequently received 4 units of packed red blood cells, stabilized and was prescribed a 10 day course of high dose progesterone for endometrial stabilization and discharged home. She was on day 6 of her treatment regimen when she presented to the same medical center again hemorrhaging heavily. This time her hemoglobin/ hematocrit was 5.9/20.7. She received 6 units of packed red blood cells and underwent a dilation and curettage. Endometrial curetting was sent to pathology with findings consistent with carcinosarcoma of the uterus. The patient underwent a computed tomography (CT) scan of the chest, abdomen and pelvis with IV contrast. There was mild right sided hydronephrosis with a sharply demarcated $5 \times 6 \mathrm{~cm}$ mass in the lower uterine segment. A pelvic ultrasound was performed, which confirmed the $6 \mathrm{~cm}$ mass within an enlarged uterus measuring $11 \mathrm{X} 8 \mathrm{~cm}$, with otherwise normal ovaries and no ascites.

The patient was transferred to a university medical center where she was placed under the care of a gynecologist oncologist. The endometrial curetting done at the transferring institution was sent to the university's pathology department to confirm malignant findings. Upon admission the patient underwent magnetic resonance imaging (MRI) of the pelvis. The report stated that there was a $6 \mathrm{~cm}$ mass that fully distended the endometrial cavity but did not appear to penetrate beyond the inner half of the myometrium with no involvement of the cervix (Figure $1 \& 2$ ). The pathology department agreed with the original diagnosis of uterine carcinomasarcoma.

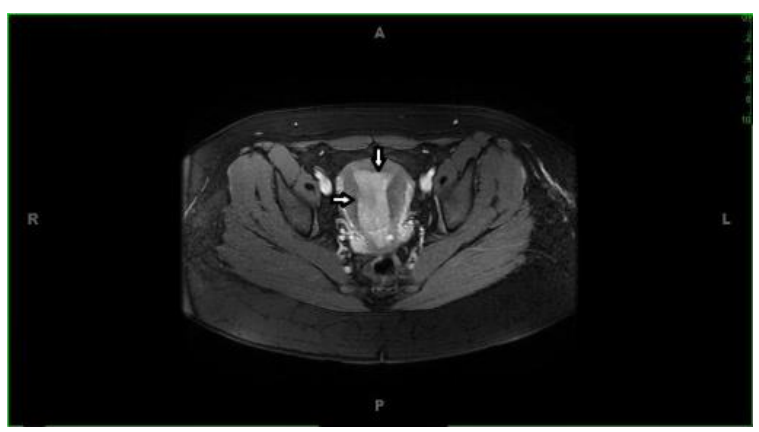

Figure 1: Axial 2D FIESTA F/S MRI of the pelvis. Arrows outlining intrauterine tumor.

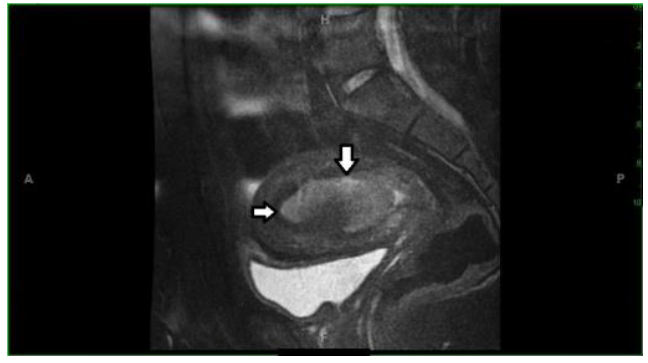

Figure 2: Sagittal T2 Weighted MRI of the pelvis. Arrows outlining intrauterine tumor.

There was extensive education at the bedside with the patient as well as her extended family on her diagnosis and management. Of course the sensitive subject for the teenage patient as well as her parents was her future fertility. She was counseled that regardless of the staging of her malignancy, there would be no possible way to spare the uterus. There was also discussion that if there was disease found involving the ovaries then they too would be removed. If the ovaries were not involved there would be a possibility of transposition of the ovaries outside of the pelvis to maintain hormonal status as well as possibility of egg retrieval with use of a surrogate carrier in the future.

Evaluation of the patient under anesthesia showed that all peritoneal surfaces were clear of disease. The uterus was enlarged with no gross disease on the surface. The ovaries as well as fallopian tubes were also normal. The patient underwent a total laparoscopic hysterectomy, bilateral salpingectomy, bilateral pelvic, para-aortic lymph node dissection, and bilateral oophoropexy. The oophoropexy in detail was accomplished by mobilizing the ovarian pedicles and transferring the ovaries outside the pelvis. With the ovaries just above the pelvic brim, they were then sutured to the abdominal sidewall within the gutters of the left and right colon. The specimens sent to pathology were uterus, cervix, bilateral fallopian tubes, two right aortic lymph nodes, three left aortic lymph nodes, ten right pelvic lymph nodes, thirteen left pelvic lymph nodes, and pelvic washings.

Pathology report on the uterine specimen was high grade carcinosarcoma with 55\% myometrial invasion. There was no involvement of any other specimen submitted including the cervix, 28 lymph nodes, and pelvic washings. According to the International Federation of Gynecology and Obstetrics (FIGO), the patient was considered stage 1B (tumor invades one half or more of the myometrium).

The patient made an uneventful postoperative recovery and was started on the Gynecological Oncology Group (GOG) Protocol 261 for chemotherapy treatment. She was started on a combination of Ifosfamide, Paclitaxel, and Mesna. During cycle \#2, she experienced a hypotensive episode with blood pressures in the 60's/40's with a maximum pulse of 172 . The reaction was 
attributed to the Paclitaxel, which was stopped. The patient had four cycles since that episode with just Ifosfamide and Mesna, and has tolerated them well. A followup CT scan done three and six months after the definitive surgery, showed no lymphadenopathy, no new infiltrates or masses.

\section{DISCUSSION}

We present an exceedingly rare case of uterine carcinosarcoma in a teenager. On a literature search using PubMed as well as Ovid, using search terms, "Uterine Carcinosarcoma", "Mixed Mullerian Tumor", "Teenager", and "Child", identified only one other case published in a medical journal of a child of 17 years with uterine carcinosarcoma. ${ }^{14}$

The management of this particular patient was challenging from the standpoint that she was only 19 and desired to keep her fertility. Oncologists treating young female patients with cancer should be aware of two important issues; first, the effect of cancer treatment on the patient's fertility and second, the available options to preserve ovarian function and chances of future pregnancy. ${ }^{15}$ To date, there are no national consensus guidelines that have been established for the management of uterine carcinsarcoma in the pre or post menopausal patient. $^{12}$ The primary treatment remains surgery; however, high rates of relapse and metastases postoperatively necessitate effective adjuvant therapies. Despite advances in adjuvant therapy, the past four decades have not seen any measurable improvement in survival. ${ }^{10}$ A major concern about ovarian transposition is the potential risk that ovarian tissue may harbor malignant cells. ${ }^{16}$ Patients in her situation have to be counseled extensively with the help of a multidisciplinary team, including an ethics committee and the team of physicians caring for the patient. Counseling should also be offered to address emotional issues prior to any decision making for both the patient and the parents.

\section{REFERENCES}

1. N'Kanza AL, Jobanputra S, Farmer P, Lovecchio J, Yelon JA, Rudloff U. Central nervous system involvement from malignant mixed Mullerian tumor (MMMT) of the uterus. Arch Gynecol Obstet 2005;273:63-8.

2. Bosquet JS, Terstriep SA, Cliby WA, et al. The impact of multi-modal therapy on survival for uterine carcinosarcomas. Gynecol Oncol 2010;116:419-23.

3. Salazar OM, Bonfiglio TA, Patten SF, et al. Uterine Sarcomas: Natural History, Treatment and Prognosis. Cancer 1978;42:1152.
4. Ramondetta LM, Burke TW, Jhingran A, et al. A phase II trial of cisplatin, ifosfamide, and mesna in patients with advanced or recurrent uterine malignant mixed müllerian tumors with evaluation of potential molecular targets. Gynecol Oncol 2003;90:529-36.

5. Sutton G, Kauderer J, Carson LF, Lentz SS, Whitney $\mathrm{CW}$, Gallion H. Adjuvant ifosfamide and cisplatin in patients with completely resected stage I or II carcinosarcomas (mixed mesodermal tumors) of the uterus: a Gynecologic Oncology Group study. Gynecol Oncol 2005;96:630-4.

6. de Jong RA, Nijman HW, Wijbrandi TF, Reyners AK, Boezen HM, Hollema H. Molecular markers and clinical behavior of uterine carcinosarcomas: focus on the epithelial tumor component. Mod Pathol 2011;24:1368-79.

7. Arora P, Rao S, Khurana N, Talwar D, Tanwar R. Malignant mixed Mullerian tumor of broad ligament with synchronous ovarian and endometrial carcinoma: a rare association. J Cancer Res Ther 2011;7:88-91.

8. Dave KS, Chauhan A, Bhansali R, et al. Uterine Carcinosarcomas: 8-year Single Center Experience of 12 Cases. Indian $\mathrm{J}$ Med Paediatr Oncol 2011;32:149.

9. Callister M, Ramondetta LM, Jhingran A, et al. Malignant Mixed Mullerian Tumors of the Uterus: Analysis of Patterns of Failure, Prognostic Factors, and Treatment Outcome. Int J Radiat Oncol Biol Phys 2004;58:786.

10. Kanthan R, Senger JL. Uterine Carcinosarcomas (Malignant Mixed Mullerian Tumours): A Review with Special Emphasis on the Controversies in Management. Obstet Gynecol Int 2011;2011:470795.

11. El-Nashar SA, Mariani A. Uterine carcinosarcoma. Clin Obstet Gynecol 2011;54:292-304.

12. Genever AV, Abdi S. Can MRI predict the diagnosis of endometrial carcinosarcoma? Clin Radiol 2011;66:621-4.

13. Lacour RA, Euscher E, Atkinson EN, et al. A phase II trial of paclitaxel and carboplatin in women with advanced or recurrent uterine carcinosarcoma. Int $\mathbf{J}$ Gynecol Cancer 2011;21:517-22.

14. Rouas L, Regragui A, Oukabli M, Amrani M, Gamra L, Otmani A, Bellabas MA. Uterine Carcinosarcoma in a Teenager: Report of a Case Macroscopically Mimicking Rhabdomyosarcoma. Rev Med Liege 2005;60:911-4.

15. Husseinzadeh N, Husseinzadeh H. Preservation of Fertility in Female Cancer Patients Desiring Future Child Bearing; What is Available and What can be Offered. World J Oncol 2013;4:1-7.

16. Sonmezer M, Shamonki MI, Oktay K. Ovarian tissue cryopreservation: benefits and risks. Cell Tissue Res 2005;322:125-132.

DOI: $10.5455 / 2320-1770 . i j r \operatorname{cog} 20130623$

Cite this article as: Atkinson AL, Osial P, Manuel

M. A 19 year old with heavy vaginal bleeding; unlikely diagnosis of uterine carcinsarcoma. Int $\mathrm{J}$ Reprod Contracept Obstet Gynecol 2013;2:221-3. 